\title{
Inpatient violence: Take steps to reduce your risk
}

\author{
Kaustubh G. Joshi, MD
}

npatient violence is a significant problem for psychiatric facilities because it can have serious physical and psychological consequences for both staff and patients. ${ }^{1}$ Victimized staff can experience decreased productivity and emotional distress, while victimized patients can experience disrupted treatment and delayed discharge. ${ }^{1}$ Twenty-five to $35 \%$ of psychiatric inpatients display violent behavior during their hospitalization. ${ }^{1}$ A subset are extreme offenders. ${ }^{1,2}$ This small group of violent patients accounts for the majority of inpatient violence and the most serious injuries. ${ }^{1,2}$

Reducing inpatient violence starts with conducting a targeted violence risk assessment to identify patients who are at elevated risk of being violent. Although conducting a targeted violence risk assessment is beyond the scope of this article, here I outline practical steps that clinicians can take to reduce the risk of inpatient violence. These steps complement and overlap with those I described in "Workplace violence: Enhance your safety in outpatient settings" (Pearls, Current Psychiatry, August 2021, p. 37-38). These approaches should be customized for your setting with the possible assistance and input of legal counsel, risk management, and law enforcement. ${ }^{3}$

Identify underlying motives. Inpatient violence is often a result of 3 primary psychiatric etiologies: difficulty with impulse control, symptoms of psychosis, or predatory traits. ${ }^{1}$ Impulsivity drives most of the violence on inpatient units, followed by predatory violence and symptoms of psychosis. ${ }^{1}$ Once you identify the psychiatric motive, you can develop an individualized, tailored treatment plan to reduce the risk of violence. The treatment plan can include using de-escalation techniques, administering scheduled and as-needed medications to target underlying symptoms, having patients assume responsibility for their behaviors, holding patients accountable for their behaviors, and other psychosocial interventions. ${ }^{1}$ Use seclusion and restraint only when it is the least restrictive means of providing safety. ${ }^{1,4}$

Develop plans and policies. As you would do in an outpatient setting, assess for hazards within the inpatient unit. Plan for the possible types of violence that may occur on the unit (eg, physical violence against hospital personnel and/or other patients, verbal harassment, etc). ${ }^{3}$ Develop policies and procedures to identify, communicate, track, and document patients' concerning behaviors (eg, posting a safety board where staff can record aggressive behaviors and other safety issues). ${ }^{3,4}$ When developing these plans and policies, include patients by creating patient/staff workgroups to develop expectations for civil behavior that apply to both patients and staff, as well as

\section{Every issue of CURRent PsYChiATRY} has its 'Pearls'

\section{Yours could be found here.}

Read the 'Pearls' guidelines for manuscript submission at MDedge.com/ CurrentPsychiatry/page/pearls. Then, share with your peers a 'Pearl' of wisdom from your practice.
Dr. Joshi is Associate Professor of Clinical Psychiatry and Associate Director, Forensic Psychiatry Fellowship, Department of Neuropsychiatry and Behavioral Science, University of South Carolina School of Medicine, Columbia, South Carolina.

\section{Disclosure}

The author reports no financial relationships with any companies whose products are mentioned in this article, or with manufacturers of competing products.

doi: 10.12788/cp.0201 
Frequently ask staff if they have any safety concerns, and solicit their opinions on how to reduce risks training patients to co-lead groups dealing with accepting responsibility for their own recovery. ${ }^{5}$ These plans and policies should include informing patients that threats and violence will not be tolerated. Frequently review these plans and policies with patients and staff.

Provide communication and education. Maintain strong psychiatric leadership on the unit that encourages open lines of communication. Encourage staff to promptly report incidents. Frequently ask staff if they have any safety concerns, and solicit their opinions on how to reduce risks. ${ }^{4}$ Include discussions about safety during staff and community meetings. Communicate patients' behaviors that are distressing or undesired (eg, threats, harassment, etc) to all unit personnel. ${ }^{3}$ Notify staff when you plan to interact with a patient who is at risk for violence or is acutely agitated. ${ }^{4}$ Teach staff how to recognize the nonverbal warning signs of behavior escalation and provide training on proper de-escalation and response. ${ }^{3,4}$ Also train staff on how to develop strong therapeutic alliances with patients. ${ }^{1}$ After a violent incident, use the postincident debriefing session to gather information that can be used to develop additional interventions and reduce the risk of subsequent violence. ${ }^{1}$

Implement common-sense strategies. Ensure that there are adequate numbers of nursing staff during each shift. ${ }^{1}$ Avoid overcrowded units, hallways, and common areas. Consider additional monitoring during unit transition times, such as during shift changes, meals, and medication administration. ${ }^{1}$ Avoid excessive noise. ${ }^{1}$ Employ one-to-one staff observation as clinically indicated. ${ }^{1}$ Avoid taking an authoritarian stance when explaining to patients why their requests have been denied ${ }^{4}$; if possible, when you are unable to meet a patient's demands, offer them choices. ${ }^{1,4}$ If feasible, accompany patients to a calmer space where they can de-escalate. ${ }^{1}$ Install video surveillance cameras at entrances, exits, and other strategic locations and post signs signaling their presence. ${ }^{3}$ Install panic buttons at the nursing station and other areas (eg, restrooms). ${ }^{3}$

Ensure your personal safety. As mentioned previously, do not interact with a patient who has recently been aggressive or has voiced threats without adequate staff support. ${ }^{4}$ During the patient encounter, leave space between you and the patient. ${ }^{1}$ Avoid having your back to the exit of the room, ${ }^{3,4}$ and make sure the patient is not blocking the exit and that you can leave the room quickly if needed. Don't wear anything that could be used as a weapon against you (eg, ties or necklaces) or could impede your escape. ${ }^{4}$ Avoid wearing valuables that might be damaged during a "take down." ${ }^{\prime 4}$ If feasible, wear an audible alarm. ${ }^{3}$

\section{References}

1. Fisher K. Inpatient violence. Psychiatr Clin North Am 2016;39(4):567-577.

2. Kraus JE, Sheitman BB. Characteristics of violent behavior in a large state psychiatric hospital. Psychiatr Serv. 2004;55(2):183-185.

3. Neal D. Seven actions to ensure safety in psychiatric office settings. Psychiatric News. 2020;55(7):15.

4. Xiong GL, Newman WJ. Take CAUTION in emergency and inpatient psychiatric settings. Current Psychiatry. 2013;12(7):9-10

5. Hardy DW, Patel M. Reduce inpatient violence: 6 strategies. Current Psychiatry. 2011;10(5):80-81.
Discuss this article at www.facebook.com/ MDedgePsychiatry 